\title{
ORIGINAL ARTICLE Genetic dissection of intraspecific variation in a male-specific sexual trait in Drosophila melanogaster
}

\author{
KM Cloud-Richardson ${ }^{1}$, BR Smith ${ }^{1}$ and SJ Macdonald ${ }^{1,2}$
}

An open question in evolutionary biology is the relationship between standing variation for a trait and the variation that leads to interspecific divergence. By identifying loci underlying phenotypic variation in intra- and interspecific crosses we can determine the extent to which polymorphism and divergence are controlled by the same genomic regions. Sexual traits provide abundant examples of morphological and behavioral diversity within and among species, and here we leverage variation in the Drosophila sex comb to address this question. The sex comb is an array of modified bristles or 'teeth' present on the male forelegs of several Drosophilid species. Males use the comb to grasp females during copulation, and ablation experiments have shown that males lacking comb teeth typically fail to mate. We measured tooth number in $>700$ genotypes derived from a multiparental advanced-intercross population, mapping three moderate-effect loci contributing to trait heritability. Two quantitative trait loci (QTLs) coincide with previously identified intra- and interspecific sex comb QTL, but such overlap can be explained by chance alone, in part because of the broad swathes of the genome implicated by earlier, low-resolution QTL scans. Our mapped QTL regions encompass 70-124 genes, but do not include those genes known to be involved in developmental specification of the comb. Nonetheless, we identified plausible candidates within all QTL intervals, and used RNA interference to validate effects at four loci. Notably, TweedleS expression knockdown substantially reduces tooth number. The genes we highlight are strong candidates to harbor segregating, functional variants contributing to sex comb tooth number.

Heredity (2016) 117, 417-426; doi:10.1038/hdy.2016.63; published online 17 August 2016

\section{INTRODUCTION}

Understanding the evolutionary and developmental basis of novel morphological, physiological and behavioral traits is a critical problem in genetics. Such traits can represent fundamental differences between closely related species, and dissecting their genetic and developmental emergence can help to uncover the evolutionary processes that occur during species divergence. In combination with elucidating the genetic basis of phenotypic differences between species, it is additionally essential to characterize the variation that segregates within natural populations, and articulate the relationship between inter- and intraspecific trait variation. One of the oldest debates in evolutionary biology is over the forces that maintain standing phenotypic variation within species (Provine, 1971; Lewontin, 1974). One set of models posit that variation is largely a result of intermediate-frequency polymorphisms that are actively maintained in populations by balancing selection (Barton and Keightley, 2002; Turelli and Barton, 2004). These models are consistent with Darwin's idea that variation between species is simply an extension of preexisting variation segregating within populations (Darwin, 1859; Lewontin, 1974). An alternative series of models propose that the bulk of variation is due to continuously arising, individually rare deleterious mutations (Johnson and Barton, 2005). If true, species diversification occurs by the rapid fixation of highly infrequent spontaneous advantageous mutations, such that there are qualitative differences between variants leading to species differences and variants that segregate within species. One effective way to determine the evolutionary processes acting on complex trait variation is to experimentally identify and characterize the underlying molecular genetic basis of multiple quantitative trait loci (QTLs), identify the genes involved and ultimately detect the molecular signatures of specific selective forces at functional loci (Mitchell-Olds et al., 2007).

Male secondary sexual traits-courtship displays, body coloration, ornaments, armaments/weapons and so on-provide abundant examples of evolutionary novelty, with many instances of rapidly evolving traits discriminating closely related species (Eberhard, 1985; Andersson, 1994). Evolution of these traits is ultimately because of competition for reproduction, driven by sexual selection on variation in male traits via processes such as female choice, male-to-male competition and sexual conflict (Parker, 1979; Andersson, 1994; Arnqvist, 1998; Hosken and Stockley, 2004; Emlen et al., 2005; Emlen, 2008). By studying the genetic control of male-specific secondary sexual traits within and between species, we can learn about the processes and selective forces leading to diversification.

In Drosophila one such male-limited secondary sexual trait is the sex comb, a cluster of specialized bristles present on the forelegs of some species within the melanogaster and obscura groups (Kopp and True, 2002; Kopp, 2011). Among closely related species within these clades, sex combs vary radically in the number of 'teeth' on each comb, the morphology of the teeth, the orientation of the comb relative to the leg axis and the position and number of comb arrays on the forelegs (Kopp and True, 2002; Barmina and Kopp, 2007; Kopp, 2011).

${ }^{1}$ Department of Molecular Biosciences, University of Kansas, Lawrence, KS, USA and ${ }^{2}$ Center for Computational Biology, University of Kansas, Lawrence, KS, USA Correspondence: Dr SJ Macdonald, Department of Molecular Biosciences, University of Kansas, 1200 Sunnyside Avenue, Lawrence, KS 66045, USA. E-mail: sjmac@ku.edu

Received 27 February 2016; revised 7 June 2016; accepted 22 June 2016; published online 17 August 2016 
The function of the sex comb has been examined in a number of studies that have physically and genetically ablated the entire comb, or experimentally reduced the number of teeth on each comb, to assess the behavior of manipulated males and their ability to copulate. Early studies showed reduced copulation/insemination success in Drosophila mauritiana, D. persimilis, D. pseudoobscura and D. simulans males that had forelegs severed to remove the region carrying the sex comb (Spieth, 1952; Coyne, 1985), and in D. melanogaster and D. simulans males where comb teeth were physically removed with forceps (Cook, 1977). A reduction in the mating success of combless D. melanogaster males was also observed by Ng and Kopp (2008) using genetic ablation of the sex comb. Recently, an elegant study employed precise laser ablation of comb teeth in both D. melanogaster and $D$. bipectinata, finding that the removal of all comb teeth in either species effectively eliminates copulation in both no choice and competitive trials (Hurtado-Gonzales et al., 2015). The failure of males to copulate in these experiments appears to be because of the inability of the male to grasp the female's abdomen/genitalia as a result of the absence of the sex comb teeth (Hurtado-Gonzales et al., 2015).

If removal of all comb teeth is deleterious to males, the question arises of whether the precise number of teeth that a male possesses is related to function, and is under selection. Surveys of several populations and strains of $D$. melanogaster show that males generally have 7-14 teeth per comb (Ahuja and Singh, 2008; Sharma et al., 2011; Snook et al., 2013). Ahuja and Singh (2008) employed divergent artificial selection and generated high and low sex comb tooth populations. Competing pairs of males with differing tooth numbers (means of 5.45 and 3.31 teeth) from a low-selected strain revealed that those with more teeth were significantly more likely to achieve a successful copulation, suggesting sexual selection against very low sex comb tooth number (Ahuja and Singh, 2008). Nevertheless, an experiment that maintained D. melanogaster and D. pseudoobscura populations for many generations under regimes allowing various levels of sexual selection failed to lead to any correlated selection for differences in sex comb tooth number (Snook et al., 2013). Studies to assess the level of sexual selection acting on the sex comb in natural, wild-caught populations (by counting teeth on the sex combs of males captured while copulating or not) have shown similarly mixed outcomes. Markow et al. (1996) found that D. simulans with relatively fewer teeth have greater copulation success. Conversely, Polak et al. (2004) found that $D$. bipectinata had greater mating success when the number of teeth on one of the three comb tooth rows was higher. No evidence for an effect of the number of teeth on mating was evident for D. pseudoobscura (Markow et al., 1996). Thus, although the comb as a whole appears to be critical for male mating, the relationship between mating success and the precise number of comb teeth is not yet clear.

The sex comb has become a valuable developmental genetics model for the rapid evolution of trait variation because of its exceptional morphological diversity among related species (Kopp, 2011). The diversity of comb morphology throughout the melanogaster and obscura groups is strongly related to the expression of the HOX transcription factor Sex combs reduced (Scr). Scr is upregulated in the sex comb precursor cells, often showing sexually dimorphic expression in species with sex combs (higher $\mathrm{S} c r$ expression in males), with no such increased expression or dimorphism in species lacking combs (Barmina and Kopp, 2007). Scr serves to activate doublesex $(d s x)$ in the tarsal region during the late larval stage, and $d s x$ expression subsequently initiates sex-specific development of the comb, and defines its morphology (Tanaka et al., 2011). The role of $d s x$ in the morphogenesis of the sex comb appears to be at least partly carried out through a repression of dachshund (dac) as Dac is absent from the sex comb during pupal development (Atallah et al., 2009, 2014). An unanswered question is whether these high-level developmental regulatory genes that are responsible for development of the sex comb also segregate for allelic variation that confers intraspecific variation in sex comb morphology.

In this study we sought to map loci contributing to variation in sex comb tooth number variation in the model $D$. melanogaster system. Our goal was to ask whether genomic regions contributing to intraspecific trait variation were consistent with those for interspecific variation mapped in prior studies. In addition, we ask whether genes involved in the developmental specification of the comb are also involved in intraspecific variation in comb tooth number. Using genotypes derived from the Drosophila Synthetic Population Resource (DSPR) (King et al., 2012b), a large set of recombinant inbred lines (RILs) derived from a multiparental advanced-generation intercross, we were able to map QTLs with greater resolution than in previous genome-wide studies (True et al., 1997; Macdonald and Goldstein, 1999; Nuzhdin and Reiwitch, 2000; Kopp et al., 2003; Tatsuta and Takano-Shimizu, 2006). The three QTLs we map all make small but significant contributions to trait heritability, and our data suggest that these loci may each harbor a series of alleles affecting phenotype. The overlap we see between our QTLs and those mapped in interspecific crosses can be explained by chance alone, and no mapped QTL overlaps with a priori developmental candidate genes (for example, $d a c, d s x, S c r)$. We functionally test at least one gene under each mapped QTL using tissue-specific RNA interference (RNAi), validating effects at the genes disco-r, poly $U$ binding factor $68 \mathrm{kD}$ ( $p U f 68$ ), scribbled (scrib) and TweedleS (TwdlS). These genes are strong candidates to harbor functional polymorphisms that causally affect sex comb tooth number variation within $D$. melanogaster, and provide a set of plausible candidate loci for future functional and behavioral testing.

\section{MATERIALS AND METHODS}

\section{QTL mapping population and phenotyping}

We mapped QTLs contributing to sex comb tooth number variation using genotypes derived from the DSPR (FlyRILs.org; King et al., 2012b). The DSPR consists of two sets of RILs ( $\mathrm{pA}$ and $\mathrm{pB}$ ), each descended from an advancedgeneration intercross of eight founder lines, seven specific to a panel (A1-A7 or B1-B7) and one common to both panels (AB8). Each recombinant population was maintained as a pair of replicate subpopulations (pA1, pA2, pB1, pB2) at large population size for 50 generations to expand the genetic map. RILs were subsequently established from each subpopulation via full-sib mating, and genotyped by restriction site-associated DNA sequencing (Baird et al., 2008). Using full resequencing data for the 15 founder lines and hidden Markov model we assign to each region in each RIL probabilities that the genomic segment is derived from each of the eight founders. For $95 \%$ of all positions over all RILs the most likely founder has a probability of $>0.95$, allowing accurate inference of the mosaic founder haplotype structure of each RIL. King et al. (2012b) provide full details of the development and properties of the DSPR RILs.

For the current experiment we generated and assayed the male $\mathrm{F}_{1}$ progeny of crosses between independent pairs of $\mathrm{pA}$ and $\mathrm{pB}$ RILs, in each case crossing $\mathrm{pA}$ RIL females to pB RIL males. Given the unidirectional nature of these crosses, and our use of male offspring, we are only able to address $\mathrm{X}$-linked variation segregating in the $\mathrm{pA}$ population. The crosses maintained the subpopulation structure of the DSPR by crossing pA1 to pB2 flies (subsequently referred to as 'subpopulation 1') and $\mathrm{pA} 2$ to $\mathrm{pB} 1$ flies ('subpopulation 2'), and we arbitrarily chose to cross RILs within the same subpopulation to avoid using any given RIL in more than one cross (that is, we carried out crosses $\mathrm{pA}_{1} \times \mathrm{xB} 2_{1}$ to $\mathrm{pA}_{n} \times \mathrm{pB} 2_{n}$, and crosses $\mathrm{pA} 2_{1} \times \mathrm{pB} 1_{1}$ to $\mathrm{pA}_{n} \times \mathrm{pB} 1_{n}$ ). See King et al. (2012a) for a comparison of the power and resolution of QTL mapping when 
phenotyping the $F_{1}$ progeny of RIL-by-RIL crosses versus phenotyping RILs directly.

RIL flies were allowed to lay eggs in narrow, polystyrene Drosophila vials containing cornmeal-yeast-molasses media. Flies were allowed to lay for up to 2 days, and adults were periodically cleared from vials to maintain roughly equal egg density across experimental vials. After 8-10 days, we harvested 10 virgin female flies from each pA RIL, and 10 male flies from each pB RIL under $\mathrm{CO}_{2}$ anesthesia. These flies-the parents of the experimental genotypes -were allowed to recover for at least 1 day before crossing, and flies were again allowed up to 2 days to lay eggs before being removed from vials. After 12-14 days, we collected $>10 \mathrm{~F}_{1}$ experimental males from each cross vial and stored at $-20^{\circ} \mathrm{C}$ until phenotyping. One foreleg from each test male was removed (we made no attempt to record whether we scored the left or right leg), mounted in mineral oil on a glass side and tooth number was manually scored at $\times 40$ total magnification.

We counted the number of teeth present on one sex comb for each of 10 males from each experimental cross vial. This modest within-genotype replication is because of our principal interest in estimating the mean phenotype associated with each founder haplotype in the mapping population, rather than in providing highly accurate estimates of genotype means. We scored males from 713 genotypes across 6 experimental batches, collecting data from 57 to 168 genotypes per batch. For the majority of genotypes experimental males were derived from a single experimental batch/vial. However, 56 genotypes were generated and tested as described above in more than one batch, and the correlation between means calculated separately for each batch is high (Pearson's $r=0.70, P<10^{-8}$, Supplementary File S1), giving us confidence in our phenotypes. Raw phenotype scores are presented in Supplementary File S2.

\section{Sex comb tooth number heritability}

Following the method described in Marriage et al. (2014), the broad-sense heritability of the trait was estimated separately for each subpopulation by calculating the genetic and phenotypic variance components from a linear model of the form: $Y_{i j k}=\mu+b_{i}+g_{i j}+\varepsilon_{i j k}$, where $Y_{i j k}$ is the $k$ th observation of the $j$ th genotype in the $i$ th batch, $\mu$ is the grand mean, $b_{i}$ is the random effect of batch, $g_{i j}$ is the random effect of RIL cross genotype nested within batch and $\varepsilon_{i j k}$ is the error term. The components were calculated in R (http://www.R-project. org) using the lme and VarCorr functions in the nlme package (Pinheiro et al., 2011). Note that as individuals of the same genotype were typically raised in the same vial, our heritability estimates will inevitably include some environmental effects. Heritability of the $\mathrm{pA} \times \mathrm{pB}$ cross progeny genotype means used for mapping was estimated as the genetic variance component divided by the total variance of genotype means.

\section{QTL mapping}

The general approach to mapping QTL in the DSPR is described in detail in King et al. (2012a, b), the full analytical model is provided in King et al. (2014) and we implemented mapping routines within the DSPRqtl R package (github.com/ egking/DSPRqtl; FlyRILs.org). Briefly, we regress mean male sex comb tooth number for each genotype on the 16 additive probabilities that correspond to the probabilities that maternal RIL is derived from each of the eight pA founders, and the probabilities that paternal RIL is derived from each of the eight $\mathrm{pB}$ founders. We additionally include subpopulation as a covariate as there is a difference between the two subpopulations in the average tooth number per genotype: subpopulation 1 mean $( \pm 1$ s.d. $)=10.79 \pm 0.565$, subpopulation 2 mean $( \pm 1$ s.d. $)=11.19 \pm 0.598$ (Figure 1). To estimate a genome-wide significance threshold for QTL identification we used 1000 permutations of the data (Churchill and Doerge, 1994). Finally, we used 3-LOD support intervals to approximate $95 \%$ confidence intervals on the true positions of causative loci, as for QTLs of modest effects mapped in the $\mathrm{pA} \times \mathrm{pB}$ cross design, typical 2-LOD drops may underestimate the appropriate interval (King et al., 2012a). Raw QTL mapping output is provided in Supplementary File S3.

\section{Sex comb QTLs mapped in prior studies}

A number of previous studies have mapped QTLs contributing to variation in male sex comb tooth number within and between Drosophila species. Nuzhdin and Reiwitch (2000) identified two X-linked QTLs segregating within a small

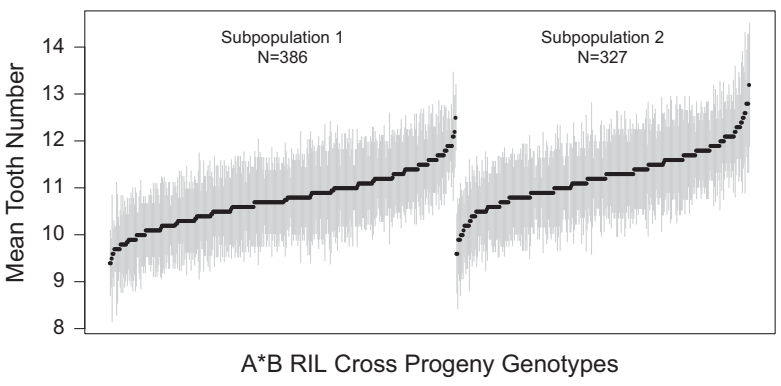

Figure 1 Variation in sex comb tooth number in the DSPR. We generated heterozygous genotypes by intercrossing 713 independent pairs of DSPR RILs, resulting in 386 genotypes from subpopulation 1 (A1 virgin females $\times$ B2 males) and 327 from subpopulation 2 (A2 virgin females $\times B 1$ males). For each genotype we counted the number of comb teeth on one leg from each of multiple male flies (mean $=11$ flies/genotype), and present the mean tooth number on a single comb for each genotype (filled circles) and 1 s.d. (vertical lines).

panel of 98 D. melanogaster RILs derived from a pair of inbred strains. Kopp et al. (2003) found eight markers associated with tooth number variation in a set of $144 \mathrm{D}$. melanogaster RILs derived from a single pair of individuals collected from nature. True et al. (1997) used a backcross mapping design to identify two QTLs contributing to the divergence between D. simulans and D. mauritiana, and in a similar study Macdonald and Goldstein (1999) found four QTLs between D. simulans and D. sechellia. Finally, Tatsuta and TakanoShimizu (2006) found five QTLs for sex comb tooth number in an $F_{2}$ cross between $D$. simulans strains.

In order to assess any overlap in QTL locations across studies we determined the position of previously mapped sex comb QTLs on our genetic map (see Supplementary File S4). When QTL positions were provided as cytological locations in these studies, we converted to nucleotide positions (D. melanogaster reference genome release 5) using the map conversion tools on FlyBase (dos Santos et al., 2015). When the QTL positions were given as the intervals between gene-based markers, we simply used the known positions of these genes from FlyBase. Nucleotide positions were subsequently converted to genetic positions on the DSPR map by virtue of the high-density genotyping conducted on all DSPR RILs, and accurate estimation of genetic distances throughout the genome (King et al., 2012a, b).

We note that unlike the other four studies, Kopp et al. (2003) used a markerbased rather than an interval-based mapping methodology and provide only the positions of significant markers. Given the haplotype structure of the mapping population, 95\% confidence intervals on QTL locations are likely to be substantially larger than reported.

Difficulties arose in two cases where Tatsuta and Takano-Shimizu (2006) mapped QTLs to intervals overlapping the proximal or distal boundaries of a large paracentric inversion on chromosome $3 \mathrm{R}$ that is fixed between D. melanogaster and the three species of the D. simulans clade (D. mauritiana, $D$. sechellia and $D$. simulans). The positions of this inversion were taken from Ranz et al. (2007), and for each of the D. simulans QTLs in question the equivalent region in $D$. melanogaster is represented by a pair of noncontiguous genomic segments.

\section{Functional validation of plausible candidate genes}

Assessment of the genes within mapped QTL intervals led us to functionally test effects on the sex comb of five genes using the bipartite Gal4-UAS RNAi system. To drive knockdown we used $r n$-Gal4 (Bloomington Drosophila Stock Center number 8142), an enhancer trap present just upstream of the rotund transcription unit, that expresses Gal4 at the position of the presumptive tarsus in the leg disc (St Pierre et al., 2002). In combination with the female splice isoform of the transformer (tra) gene under the control of the UAS promoter, the $r n$-Gal4 driver has been used to genetically ablate the sex comb in Drosophila males ( $\mathrm{Ng}$ and Kopp, 2008). We also employed three $d s x$-Gal4 drivers, gifts from the Baker (Ashburn, VA, USA) and Goodwin labs (Oxford, UK). These are targeted knock-ins of Gal4 into the native doublesex gene and 
express Gal4 in a pattern that generally recapitulates that of endogenous $d s x$ expression (Rideout et al., 2010; Robinett et al., 2010; Pan et al., 2011). Regulation of $d s x$ expression is critical for the proper development of the sex comb (Tanaka et al., 2011).

Transgenic RNAi Project (TRiP) UAS-RNAi and co-isogenic control strains were obtained from the BDSC (Bloomington, IN, USA), specifically stock numbers 36303 (chromosome 3 landing site control strain), 35786 (UAS-GFP control), 35788 (UAS-Luciferase control), 41683 (UAS-disco-r-RNAi), 31960 (UAS-Dsp1-RNAi), 25951 and 34785 (UAS-pUf68-RNAi), 29552 (UAS-scribRNAi) and 61864 (UAS-TwdlS-RNAi). We also obtained UAS-RNAi stocks, and the appropriate control strains, from the Vienna Drosophila Resource Center (Vienna, Austria) (Dietzl et al., 2007); 20144 (UAS-pUf68-RNAi), a 'GD' line harboring a $P$-element transgene, 109796 (UAS-pUf68-RNAi) and 105414 (UAS-scrib-RNAi), 'KK' lines harboring phiC31-based UAS transgenes, 60000 ( $w^{1118}$ host strain for GD library) and 60100 (KK landing site control strain). All Gal4 and UAS transgenes are inserted into autosomal locations (see Supplementary File S5 for additional details.)

We generated RNAi knockdown/control male genotypes by crossing 10 virgin females from each UAS/control strain to 10 males from each Gal4 strain, establishing multiple vials per cross over multiple blocks. For each experimental male, the phenotype was scored as the average number of teeth over both sex combs (raw phenotype scores are presented in Supplementary File S6). The final sample size for each genotype varies because of marked differences in the number of males of the desired genotype emerging from each cross vial, likely a consequence of pre-adult lethality for some of the knockdown genotypes generated. Every experimental block included control genotypes to assess any block-to-block variation in phenotype.

\section{Fly rearing}

All stock maintenance and rearing of experimental individuals was carried out at $25^{\circ} \mathrm{C}$ and $50 \%$ relative humidity with a $12 \mathrm{~h}$ light $/ 12 \mathrm{~h}$ dark cycle.

\section{Statistical analysis}

All statistics were carried out using core, or custom written, routines in the $\mathrm{R}$ statistical programming language (http://www.R-project.org).

\section{RESULTS}

\section{Heritability}

Variation in male sex comb tooth number is substantial in the DSPR (Figure 1), with heterozygous $\mathrm{pA} \times \mathrm{pB}$ cross progeny genotype means ranging from 9.4 to 13.2 teeth per sex comb. This variation is similar to the range of 9.2-12.9 teeth observed across 32 lines of D. melanogaster derived from a worldwide set of collection sites (Ahuja and Singh, 2008), and similar to the 7-13 teeth (Sharma et al., 2011) and $\sim 11$ teeth (Snook et al., 2013) present in laboratory-adapted outbred populations. On average, genotype means in subpopulation 1 are lower than those in subpopulation 2 (Welch's $t=-9.1, P<10^{-15}$ ). Given the subpopulations are derived from the same sets of founders, but were maintained independently for 50 generations before the creation of RILs, differences most likely reflect variation in the founder composition of the subpopulations (see King et al., 2012b).

The broad-sense heritability for sex comb tooth number was estimated as 0.23 and 0.22 for subpopulations 1 and 2, respectively. Variance due to batch was $<1 \%$ in each case. These heritability values are somewhat higher than, although not inconsistent with, narrowsense heritability estimates of $0.07-0.21$ from the response to artificial selection for increased and decreased comb tooth number in D. melanogaster (Ahuja and Singh, 2008). For QTL mapping we employ genotype means, averaging over all 10 sex comb measurements per genotype, and the broad-sense heritability of these mean phenotypes is 0.77 and 0.75 for subpopulations 1 and 2, respectively. The effect of environmental variation on sex comb tooth number has been reported to be much greater than that of genetic variation, with genetic and environmental coefficients of variation of 2.96-3.36 and 7.87-7.99, respectively (Ahuja and Singh, 2008). We see comparable values in this study (genetic coefficient of variation $=4.5$, environmental coefficients of variation $=8.2$ ), and hence by measuring multiple individuals we reduce the effect of environmental noise, and our mean sex comb phenotype has much higher heritability.

\section{Sex comb QTLs}

Using a similar approach to recent studies using the DSPR (Kislukhin et al., 2013; King et al., 2014) we mapped QTL contributing to variation in sex comb tooth number across $713 \mathrm{pA} \times \mathrm{pB}$ cross progeny male genotypes. Three QTLs survived a permutation-derived 5\% genome-wide threshold (Figure 2 and Table 1); Q1 is X-linked, and Q2 and Q3 are near the opposite ends of chromosome 3 on $3 \mathrm{~L}$ and 3R, respectively. QTLs explain 4.4-7.4\% of the genetic variation for sex comb tooth number and-assuming additivity-collectively explain $18.4 \%$ of the total genetic variation for the trait (Table 1). Given the fairly large sample size employed, we do not expect substantial upward bias in QTL effect estimates-the Beavis effect (Beavis, 1994; Xu, 2003)—nonetheless, the values we estimate should be considered as upper bounds on the variance explained by these loci.

Standard QTL mapping studies attempt to identify causative loci that segregate between a pair of parental genotypes (see Mackay, 2001). Thus, only a tiny fraction of the allelic variation in a population is interrogated, and such studies cannot evaluate the extent to which causative loci harbor a series of functional alleles with different effects on phenotype. With the DSPR we have the opportunity to evaluate the phenotypic effects of up to 15 alleles. Figure 3 shows the estimated founder haplotype effects at each of the mapped QTLs, and does not suggest a clear grouping of the founders into two groups, the pattern expected under simple biallelism. Although the patterns we observe could be because of allelic heterogeneity at single causative genes under each QTL, given the size of the implicated intervals (0.61-0.99 megabases; Table 1), it is not unlikely that multiple linked genes contribute to the effects estimated at each QTL. In addition, the variation in founder genotype frequency in the DSPR, where the frequency of each founder deviates from the expected $1 / 8$ throughout the genome (King et al., 2012b), could make effects difficult to estimate and complicate their interpretation.

\section{Overlap of sex comb QTLs among studies}

Two previous studies have mapped QTL for sex comb tooth number in small panels of D. melanogaster RILs (Nuzhdin and Reiwitch, 2000; Kopp et al., 2003). The positions of these QTLs are depicted in the top panel of Figure 2 (blue bars, rows $a$ and $b$ ). One of the two X-linked QTLs mapped in Nuzhdin and Reiwitch (2000) (Figure 2, row a) overlaps with Q1 mapped here, but otherwise none of the QTLs previously observed to contribute to sex comb tooth number variation within D. melanogaster overlap those we map in the DSPR. As $9.2 \%$ of the physical genome harbors previously mapped intraspecific QTL, finding one $1-\mathrm{Mb}$ DSPR QTL that overlaps such a region is not unexpected by chance (Poisson probability, $k=1, \lambda=0.092, P=0.09$ ). We see the same result when considering that previously mapped intraspecific QTLs encompass $12.9 \%$ of the genetic map length $(P=0.11)$. True differences in the architecture of trait variation among the three $D$. melanogaster mapping populations could obviously explain this lack of overlap, and clearly studies initiated from 2 to 4 alleles (Nuzhdin and Reiwitch, 2000; Kopp et al., 2003) may not have captured functional alleles present in the DSPR. Differences in power among the studies could also plausibly explain the differences we see. In this context, previous simulations of the 


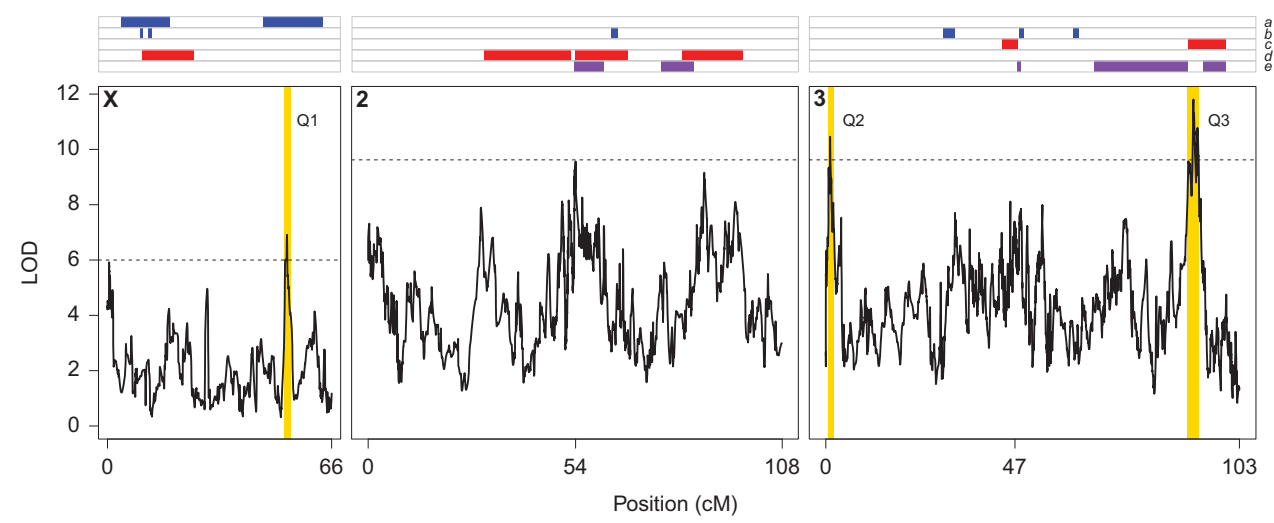

Figure 2 Genome scan for QTLs contributing to sex comb tooth number variation. The bottom three panels show a genome scan in the DSPR for sex comb tooth number QTLs (solid curve) along each of the major fly chromosomes. Genetic positions 54 and 47 on chromosomes 2 and 3, respectively, represent the positions of centromeres. The horizontal dashed lines represent genome-wide $5 \%$ permutation thresholds ( $X: L O D=6.0$, autosomes: $L O D=9.6)$. The $3-L O D$ drop intervals implicated by the three QTLs we map (Q1, Q2, Q3) are highlighted as vertical yellow bars. The top panel shows the positions of sex comb QTLs mapped in five previous studies: a, within D. melanogaster (blue; Nuzhdin and Reiwitch, 2000); b, within D. melanogaster (blue; Kopp et al., 2003); c, between D. simulans and D. mauritiana (red; True et al., 1997); d, between D. simulans and D. sechellia (red; Macdonald and Goldstein, 1999); e, within $D$. simulans (purple; Tatsuta and Takano-Shimizu, 2006). In placing the QTLs mapped in studies $c-e$ on our map we have accounted for the large chromosome $3 R$ paracentric inversion that is fixed between $D$. melanogaster and $D$. mauritianal $D$. sechellial $D$. simulans (see Materials and methods).

Table 1 Details of QTLs mapped for sex comb tooth number variation in the DSPR

\begin{tabular}{|c|c|c|c|c|c|c|}
\hline Name & Chr & $\angle O D$ score & Peak $C M(3-L O D C I)^{a}$ & Peak $M b(3-L O D C l)^{a}$ & Number of genes ${ }^{b}$ & Percent of $\mathrm{H}^{2 \mathrm{c}}$ \\
\hline Q1 & $x$ & 6.9 & $53.0(52.3-54.2)$ & $16.05(15.81-16.42)$ & 70 & 4.4 \\
\hline Q2 & $3 L$ & 10.5 & $1.1(0.6-1.9)$ & $1.48(1.22-1.90)$ & 124 & 6.6 \\
\hline
\end{tabular}

Abbreviations: Cl, confidence interval; Chr, chromosome; DSPR, Drosophila Synthetic Population Resource; LOD, logarithm (base 10) of odds; QTL, quantitative trait locus.

a3-LOD Cl indicates the 3-LOD support interval of the QTLs. Physical positions are given based on release 5 of the Drosophila reference genome.

bThe number of protein-coding genes in the 3-LOD support interval.

CThe percentage of broad-sense heritability of genotype means explained by the QTLS.

power of the DSPR $\mathrm{pA} \times \mathrm{pB}$ cross design to identify QTLs contributing $5-10 \%$ of the variation is $68-95 \%$ (King et al., 2012a). Thus, if the genetic architecture of sex comb tooth number is dominated by smallto modest-effect QTLs, insufficient power may lead to studies routinely identifying different subsets of QTLs.

Two studies have previously mapped between-species variation for sex comb tooth number (Figure 2, top panel, red bars, rows $c$ and $d$ ), using D. simulans-D. sechellia recombinants (Macdonald and Goldstein, 1999), and D. simulans-D. mauritiana recombinants (True et al., 1997). The largest-effect QTL mapped by True et al. (1997), positioned over the chromosome 3 centromere (Figure 2), has been further resolved by Graze et al. (2007) who implicated a small handful of genes as plausibly contributing to the difference in sex comb tooth number between $D$. simulans and D. mauritiana. Only our Q3 overlaps an interspecific sex comb QTL, mapping at the same location as the small-effect QTL identified by True et al. (1997) (Figure 2). The apparent differences in the architecture of within- and between-species variation we observe could be real or could be due to the technical differences in QTL mapping methods described above.

Collectively, across five previous studies, $53.3 \%$ (49.5\%) of the physical (genetic) map has been implicated in the genetic control of sex comb tooth number variation, and by random chance we would expect that two of the QTLs we map would overlap these regions (Poisson probability, $k=2, \lambda=0.533 / 0.495, P=0.08 / 0.07$ ), and this is what we observe. Interestingly, no previous studies have mapped a factor influencing sex comb tooth number variation to the left end of chromosome 3L where we identify QTL Q2 (Figure 2).
Candidate sex comb tooth number genes

Several genes are known to affect the specification and development of the sex comb, such as Scr (Lewis et al., 1980a, b; Barmina and Kopp, 2007; Tanaka et al., 2011), dac (Atallah et al., 2014), the sex determination pathway genes $d s x$ and transformer 2, tra2 (Baker and Ridge, 1980; Tanaka et al., 2011) and a series of genes named based on their effect on the sex comb (Additional sex combs, extra sex combs, multi sex combs, Posterior sex combs, Sex comb on midleg, Sex combs extra and super sex combs). Although some of these genes, such as $d s x$ and Scr (Graze et al., 2007), have been implicated within QTL intervals identified in previous mapping studies, none of them are present within the three QTLs we map in the DSPR (Supplementary File S7).

In the absence of classic candidate genes within the modest number of genes in our QTL intervals (Table 1), we employed two strategies to attempt to define plausible candidates for subsequent testing. First, to identify any genes in FlyBase with a putative or functionally characterized role in sex comb development, we used the controlled vocabulary search terms 'sex comb', 'sex comb tooth' and 'sex comb development' to extract a total of 93 genes tagged with one or more of these terms (see Supplementary File S8). The genes Dsp1 (Dorsal switch protein 1) and pUf68 (poly $U$ binding factor $68 \mathrm{kD}$ ) were previously annotated as having some role in sex comb formation (Decoville et al., 2001; Quinn et al., 2004), and are present within QTL intervals Q1 and Q2, respectively. Second, we exploited data from a microarray study (Barmina et al., 2005) that identified two independent sets of genes of interest (see Supplementary Tables 1 and 3 from Barmina et al., 2005): (1) 16 genes differentially expressed 

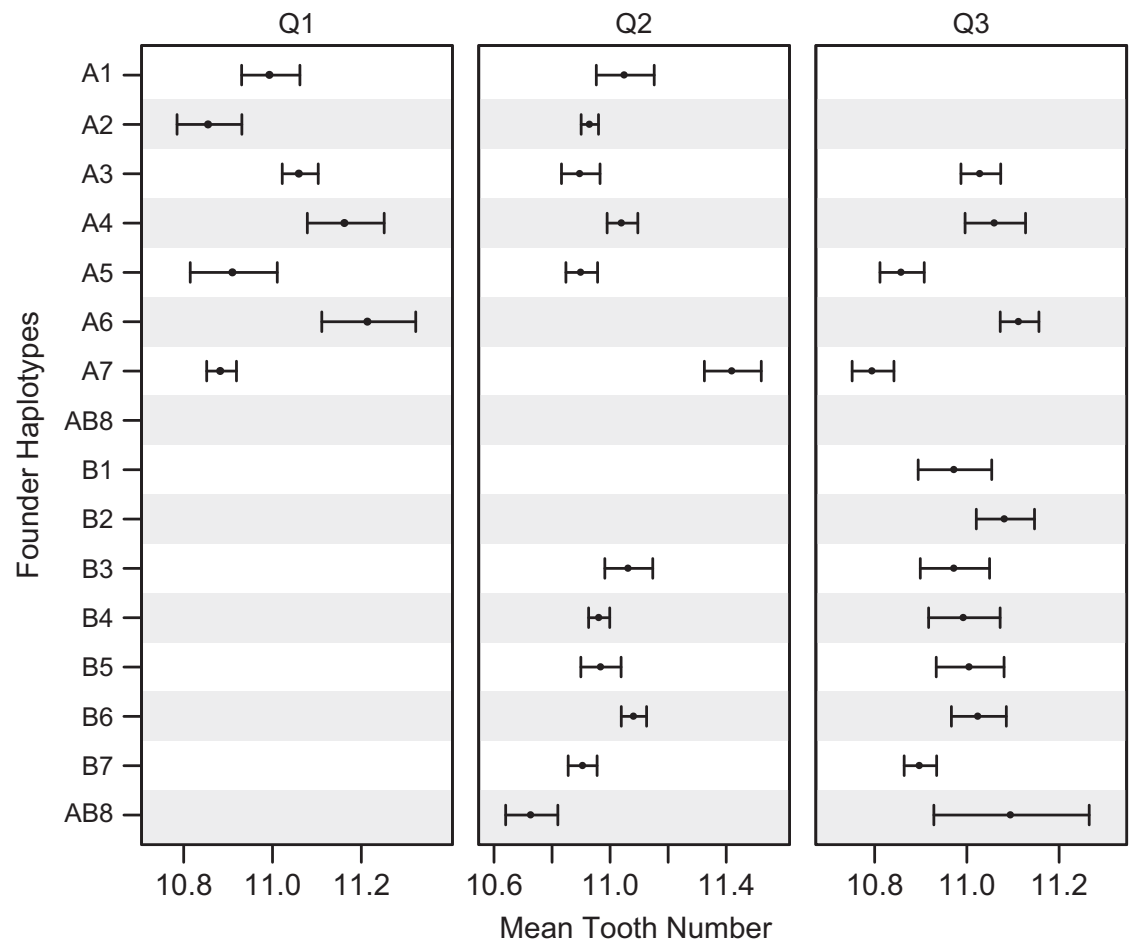

Figure 3 Founder haplotype effects for mapped QTLs. Phenotype means (and s.e.) are presented for each founder at the peak position of each QTL. Data are only presented for founders present in at least 10 RILs at a probability of $>0.95$. AB8 was used to found both synthetic populations, and hence is depicted twice in the figure. Given that we phenotyped males resulting from crosses between population A females and population B males, for the X-linked Q1 only means for population A founders can be estimated.

between male, sex comb-bearing T1 pupal legs (forelegs) and male, non-sex comb-bearing T2 pupal legs and (2) 143 genes differentially expressed between male T1 pupal legs and female, non-sex combbearing T1 pupal legs. TwdlS (TweedleS) was differentially expressed between male T1 and T2 legs, and is under Q3. Genes disco-r (disco-related) and scrib (scribbled) were differentially expressed between male $\mathrm{T} 1$ and female $\mathrm{T} 1$ legs, and are present within the Q1 and Q3 intervals, respectively.

We attempted to functionally validate the effect of these five genes on the number of sex comb tooth in D. melanogaster using the bipartite Gal4-UAS RNAi system. The two types of Gal4 drivers used — $r n$-Gal4 and $d s x$-Gal4 - are expected to knock down expression of target genes in slightly different spatiotemporal patterns, but in both cases include the presumptive sex comb precursor region of the foreleg.

Knockdown of disco-r (under Q1) shows no effect with the $r n$-Gal4 driver (Figure 4), but we see a reduction in the number of sex comb teeth with two of the three $d s x$-Gal4 drivers (Figure 5). Knockdown of Dsp1 (also under Q1) shows no effect on the phenotype with any driver (Figures 4 and 5), potentially implying it has no role in the sex comb, although we cannot discount the possibility that Dsp1 expression is simply not being reduced because of technical factors (see Booker et al., 2011).

For $p U f 68$ (under Q2) we see a significant change in phenotype for all $r n$-Gal4 knockdowns tested (Figure 4), but none of the $d s x$-Gal4 knockdowns (Figure 5). The situation is complicated by the observation that although three of the pUf68 rn-Gal4 knockdowns yield lower sex comb tooth numbers than the control, in one case (construct ID 34785 ) we see significantly more teeth (Figure 4 ). We regenerated this latter pUf68 RNAi genotype using the reciprocal cross (crossing males from the UAS strain to females from the Gal4 strain) that serves to swap the $\mathrm{X}$ chromosome present in the test males, but observed the same increase in comb tooth number relative to the control (Supplementary File S9). Given the irregular formation of the comb in pUf68 RNAi animals, and the variable size and melanization of the teeth (Supplementary File S10), some of the variations across genotypes may simply represent experimental difficulty counting the number of teeth present.

For scrib (under Q3) there is a significant reduction in sex comb tooth number with $\mathrm{rn}$-Gal4 (Figure 4), and we also observe a reduction with one of the three $d s x$-Gal4 drivers (Figure 5). For TwdlS (also under Q3) we see a dramatic reduction in sex comb tooth number across all drivers (Figures 4 and 5 and Supplementary File S10).

\section{DISCUSSION}

Darwin posited the conversion of variation within species into adaptive differences among species. If genes that contribute to polymorphism within a species are generally also those that influence a trait difference between species, there should be significant overlap between mapped intra- and interspecific QTL intervals. Several previous studies have observed QTL overlap in both within and between species (Nuzhdin and Reiwitch, 2000; Fishman et al., 2002; Lexer et al., 2005; Hall et al., 2006; Wittkopp et al., 2009; McNeil et al., 2011; Groot et al., 2013), supporting the idea that interspecific differences are not fundamentally distinct from variation within species in many instances. However, at least one study failed to identify any overlap in the positions of QTLs mapped within and between species (Gleason and Ritchie, 2004). QTL overlap does not provide information on whether those alleles responsible for species 

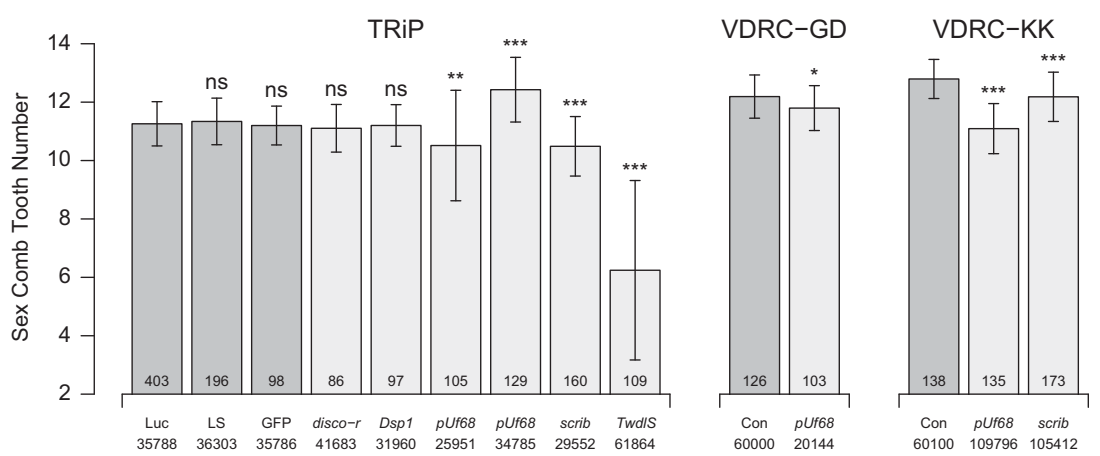

Figure $4 \mathrm{rn}$-Gal4 RNAi knockdown of plausible candidate genes implicated by QTL. Each bar represents the mean tooth number on a single comb ( $\pm 1 \mathrm{~s}$.d.) for several $r n$-Gal4/UAS male RNAi knockdown (light gray bars) and control genotypes (dark gray bars). The name of each UAS control or gene is provided beneath each bar along with the stock number of the UAS strain in the Bloomington Drosophila Stock Center (TRiP) or the Vienna Drosophila Resource Center (VDRC). The sample size per genotype is presented at the bottom of each bar. The phenotype of each knockdown was compared with the appropriate control via Welch's $t$-tests (NS, not significant, ${ }^{*} P<0.01,{ }^{*} * P<0.0005,{ }^{* * *} P<10^{-10}$ ). For TRiP lines we tested three control strains-'Luc' (UAS-Luciferase), 'LS' (landing site for phiC31-based transgenesis) and 'GFP' (UAS-GFP) - and here present statistical comparison of each gene knockdown to 'Luc'. The 'Luc' control genotype was tested in all experimental blocks, and no significant block-to-block variation was observed for this genotype. For VDRC-GD and -KK lines the control genotype is listed as 'Con'.

differentiation were present as standing variation in the ancestral populations from which the species arose, or were generated by new mutations. However, it can suggest that extant allelic variation within a species, and differences between species, reside at the same loci, demonstrating shared genetic control of trait variation within and between species.

Here, we genetically tested Darwin's idea using variation in the number of teeth on the sex comb, a male-specific sexual structure in Drosophila. The gross morphology of the sex comb has rapidly diverged among closely related species (Kopp, 2011), there is variation among the four melanogaster complex species in the number of comb teeth (True et al., 1997) and there is some evidence that tooth number is under selection (see, for example, Ahuja and Singh, 2008). Using a multiparental, advanced intercross mapping population, and phenotyping well over 7000 animals, we mapped three QTLs contributing to sex comb tooth variation within $D$. melanogaster. We subsequently examined overlap between the positions of QTLs contributing to sex comb tooth number in the DSPR, and the positions of intra- and interspecific sex comb QTLs identified in previous work using the melanogaster group of Drosophilid species (True et al., 1997; Macdonald and Goldstein, 1999; Nuzhdin and Reiwitch, 2000; Kopp et al., 2003; Tatsuta and Takano-Shimizu, 2006). The level of overlap between the QTLs that we identified and those identified in five previous mapping studies is not strong, and indeed is consistent with chance. Nonetheless, neither is overlap absent. Q1 overlaps with one of the two X-linked QTLs mapped by Nuzhdin and Reiwitch (2000) in a cross between D. melanogaster strains (Figure 2), and Q3 overlaps with a QTL mapped to the end of chromosome 3R by True et al. (1997) in a between-species mapping panel (Figure 2). It is possible we have replicated effects at the same genes in these instances, but available data preclude any confident test of this assertion. The low resolution of early QTL mapping studies, which ensures large fractions of the genome are implicated in the control of trait variation, and the imperfect power to map small-effect QTLs in the DSPR, which means only a subset of the functional polymorphisms are resolved, makes assessing the similarity of intra- and interspecific genetic architecture for sex comb tooth number technically challenging.

One biological limitation of the within- and between-species comparison we present is the lack of an interspecific cross involving D. melanogaster. Such crosses are not possible because of hybrid inviability and sterility. A more appropriate test may be to build on the work of Tatsuta and Takano-Shimizu (2006) and generate highresolution mapping data within $D$. simulans, allowing direct comparison with interspecific variation between $D$. simulans and either $D$. mauritiana or $D$. sechellia. Recent advances in high-throughput genotyping (see, for example, Andolfatto et al., 2011) allow very large mapping populations in non-model systems to be genetically analyzed (Huang and Erezyilmaz, 2015). This offers the possibility of mapping sex comb number QTL in non-D. melanogaster recombinants with sufficient resolution that the similarity of trait architecture within and between species can be compared with confidence.

In contrast to previous mapping studies, we were able to use the DSPR to map modest-effect QTL to short, statistically defined regions, providing the opportunity to uncover likely candidate genes. None of the three QTLs we map implicate dac, $d s x$, Scr or tra2 (Supplementary File S7), the four genes that have been shown to be involved in the specification and development of the sex comb (Baker and Ridge, 1980; Lewis et al., 1980a, b; Barmina and Kopp, 2007; Tanaka et al., 2011; Atallah et al., 2014). Although not formally significant at a 5\% permutation-derived threshold (Churchill and Doerge, 1994), we observed three additional peaks in the LOD (logarithm (base 10) of odds) score profile that are close to genome-wide significance (X, $0.5 \mathrm{cM}$; 2L, $54.0 \mathrm{cM}$; and 2R, $87.5 \mathrm{cM}$; Figure 2 and Supplementary File S3). These peaks could represent true, small-effect QTLs or simply be spurious. Regardless, 3-LOD support intervals about these peaks also do not implicate $d a c, d s x$, Scr or tra2. These results imply that key players in sex comb developmental patterning either do not contribute to natural variation in sex comb tooth number in the DSPR or were undetectable in our study by virtue of having relatively small individual effects or by segregating for very rare functional alleles not captured in the DSPR.

Despite the absence of a priori candidate genes beneath mapped QTL intervals, we were able to leverage other data to define one or two plausible candidates for each QTL, and used RNAi directed to the region of the foreleg from which the sex comb is derived to show that four of these genes affect sex comb tooth number. disco- $r$ is present within the interval implicated by X-linked QTL Q1, was shown to have lower expression in male compared with female pupal forelegs (Barmina et al., 2005) and has been suggested to be involved in leg development (Grubbs et al., 2013). pUf68 is implicated by QTL Q2 on 

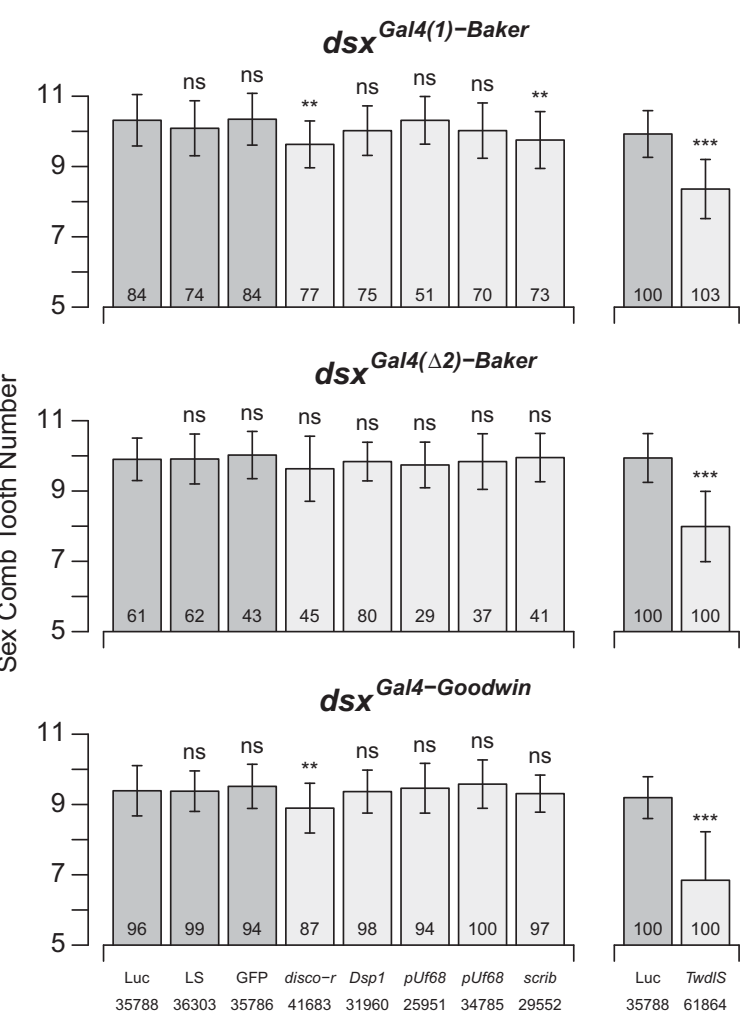

Figure $5 d s x$-Gal4 RNAi knockdown of plausible candidate genes implicated by QTLs. We used three different $d s x-G a l 4$ lines to knock down candidate gene expression; $d s x^{G a l 4(1)}$ is described in Robinett et al. (2010), $d s x^{G a l 4(\Delta 2)}$ in Pan et al. (2011) and $d s x^{G a / 4}$ in Rideout et al. (2010). The eight genotypes on the left of each panel were generated and scored in one experimental block, whereas the two genotypes on the right were generated/ scored in an independent block. Because of a slight but significant block-toblock difference in the mean phenotype of the control genotype containing UAS-Luciferase ('Luc'), knockdown genotypes were compared only with controls generated and scored contemporaneously. See Figure 4 for details of the labels and presentation of the data. NS, not significant, ${ }^{* *} P<0.0005,{ }^{* * *} P<10^{-10}$.

chromosome 3. This gene is differentially expressed between adult virgin males and females (McIntyre et al., 2006), and a loss-offunction mutation leads to malformed sex combs (Quinn et al., 2004). The product of $p U f 68$ is known to regulate alternative splicing (Van Buskirk and Schupbach, 2002), and is required for splicing of the M1 intron from the tra2 primary transcript, although it does not appear to contribute to Tra2 control of $d s x$ splicing (Wang et al., 2013). scrib is present within QTL Q3 on chromosome 3 and, similar to disco- $r$, has been shown to have lower gene expression in male forelegs compared with female forelegs (Barmina et al., 2005). This gene is a tumor suppressor involved in the control of epithelial cell proliferation (Bilder et al., 2000) and epithelial cell polarity (Bilder and Perrimon, 2000), and has been shown to regulate asymmetric cell division in Drosophila neuroblasts (Albertson and Doe, 2003). In addition, scrib was isolated during a $P$-element mutagenesis screen to identify genes showing defects in sensory bristle number (Norga et al., 2003). Finally, TwdlS is also present under Q3, and was previously shown to be differentially expressed between male T1 and T2 legs (Barmina et al., 2005). Little is known about the functional role of this gene, although based on analyses of other genes in the same family, the family is insect specific and may have a role in assembly of the cuticle (Guan et al., 2006). In our study the effect on sex comb tooth number of a knockdown of TwdlS expression was pronounced (Figures 4 and 5) and the magnitude of the phenotypic change was greater than for any other gene we tested. In addition, for all $d s x$-Gal4-driven TwdlS knockdowns, we noted that the mutant male flies appeared to completely lack proper external genitalia, suggesting the gene may play a critical, higher-order role in sexual trait determination in D. melanogaster. One caveat to these RNAi-based functional tests is the possibility for off-target, nonspecific effects of the RNAi reagents to lead to false positives (Ma et al., 2006). However, such effects should be minimized in cases where multiple transgenes yield similar results.

In summary, in this study we have used a combination of highresolution QTL mapping, published expression data and RNAi-based functional tests to implicate several short regions of the Drosophila genome, and a handful of loci, in the genetic control of male sex comb tooth number variation. Three of the candidate sex comb genes that we identify-disco-r, scrib and Twdls - have not previously been shown to affect the morphology of the sex comb, and given their reported expression differences between sexes or segments in the Drosophila foreleg (Barmina et al., 2005), may be expected to segregate for functional regulatory polymorphisms that affect sex comb tooth number. Identification of the precise causative variants may be accomplished by association mapping in natural populations of flies (see, for example, Bastide et al., 2013), although the large environmental component to the variation of sex comb tooth number, the subtle effects of the QTLs we map and the possibility that QTLs are multiallelic imply that the kinds of sample size typically employed in the human disease association mapping literature will be required (that is, tens of thousands of individuals). Alternatively, it is now becoming feasible in D. melanogaster to employ CRISPR-Cas9 genome editing to compare different, putatively functional alleles in an otherwise standardized background and directly test the effects of specific genomic intervals, genes and nucleotide positions.

\section{DATA ARCHIVING}

All phenotype data collected as part of this study are available as Supplementary Information files. All data on the DSPR project is available at FlyRILs.org.

\section{CONFLICT OF INTEREST}

The authors declare no conflict of interest.

\section{ACKNOWLEDGEMENTS}

We thank Matthew Turner and Whitney Sutton for assistance in scoring the phenotype. We are grateful to Carmen Robinett (Bruce Baker lab) and Hania Pavlou (Stephen Goodwin lab) for kindly sharing $d s x$-Gal4 knock-in lines. Stocks obtained from the Bloomington Drosophila Stock Center (NIH P40OD018537) and the Vienna Drosophila Resource Center (stockcenter.vdrc. at) were used in this study. We thank the TRiP at Harvard Medical School (NIH R01GM084947) for providing transgenic RNAi fly stocks used in this study. John Kelly provided helpful comments on an earlier version of this manuscript. This work was supported by National Institutes of Health (NIH) Grant R01 GM085260 to SJM.

Ahuja A, Singh RS (2008). Variation and evolution of male sex combs in Drosophila: nature of selection response and theories of genetic variation for sexual traits. Genetics 179: 503-509.

Albertson R, Doe CQ (2003). Dlg, Scrib and Lgl regulate neuroblast cell size and mitotic spindle asymmetry. Nat Cell Biol 5: 166-170.

Andersson M (1994). Sexual Selection. Princeton University Press: Princeton, NJ.

Andolfatto P, Davison D, Erezyilmaz D, Hu TT, Mast J, Sunayama-Morita T et al. (2011). Multiplexed shotgun genotyping for rapid and efficient genetic mapping. Genome Res 21: $610-617$. 
Arnqvist G (1998). Comparative evidence for the evolution of genitalia by sexual selection. Nature 393: 784-786.

Atallah J, Liu NH, Dennis P, Hon A, Larsen EW (2009). Developmental constraints and convergent evolution in Drosophila sex comb formation. Evol Dev 11: 205-218.

Atallah J, Vurens G, Mavong S, Mutti A, Hoang D, Kopp A (2014). Sex-specific repression of dachshund is required for Drosophila sex comb development. Dev Biol 386: 440-447.

Baird NA, Etter PD, Atwood TS, Currey MC, Shiver AL, Lewis ZA et al. (2008). Rapid SNP discovery and genetic mapping using sequenced RAD markers. PLoS One 3: e3376.

Baker BS, Ridge KA (1980). Sex and the single cell. I. On the action of major loci affecting sex determination in Drosophila melanogaster. Genetics 94: 383-423.

Barmina O, Gonzalo M, McIntyre LM, Kopp A (2005). Sex- and segment-specific modulation of gene expression profiles in Drosophila. Dev Biol 288: 528-544.

Barmina O, Kopp A (2007). Sex-specific expression of a HOX gene associated with rapid morphological evolution. Dev Bio/ 311: 277-286.

Barton NH, Keightley PD (2002). Understanding quantitative genetic variation. Nat Rev Genet 3: 11-21.

Bastide H, Betancourt A, Nolte V, Tobler R, Stobe P, Futschik A et al. (2013). A genomewide, fine-scale map of natural pigmentation variation in Drosophila melanogaster. PLoS Genet 9: e1003534.

Beavis WD (1994). The power and deceit of QTL experiments: lessons from comparative QTL studies. Proceedings of the 49th Annual Corn and Sorghum Industry Research Conference. American Seed Trade Association: Washington, DC. pp 250-266.

Bilder D, Li M, Perrimon N (2000). Cooperative regulation of cell polarity and growth by Drosophila tumor suppressors. Science 289: 113-116.

Bilder D, Perrimon N (2000). Localization of apical epithelial determinants by the basolateral PDZ protein Scribble. Nature 403: 676-680.

Booker M, Samsonova AA, Kwon Y, Flockhart I, Mohr SE, Perrimon N (2011). False negative rates in Drosophila cell-based RNAi screens: a case study. BMC Genomics 12: 50.

Churchill GA, Doerge RW (1994). Empirical threshold values for quantitative trait mapping Genetics 138: 963-971.

Cook RM (1977). Behavioral role of the sexcombs in Drosophila melanogaster and Drosophila simulans. Behav Genet 7: 349-357.

Coyne JA (1985). Genetic studies of three sibling species of Drosophila with relationship to theories of speciation. Genet Res 46: 169-192.

Darwin C (1859). The Origin of Species. John Murray: London, UK.

Decoville M, Giacomello E, Leng M, Locker D (2001). DSP1, an HMG-like protein, is involved in the regulation of homeotic genes. Genetics 157: 237-244.

Dietzl G, Chen D, Schnorrer F, Su KC, Barinova Y, Fellner M et al. (2007). A genome-wide transgenic RNAi library for conditional gene inactivation in Drosophila. Nature 448 $151-156$.

dos Santos G, Schroeder AJ, Goodman JL, Strelets VB, Crosby MA, Thurmond J et al. (2015). FlyBase: introduction of the Drosophila melanogaster Release 6 reference genome assembly and large-scale migration of genome annotations. Nucleic Acids Res 43: D690-D697.

Eberhard WG (1985). Sexual Selection and Animal Genitalia. Harvard University Press: Cambridge, MA.

Emlen DJ (2008). The evolution of animal weapons. Annu Rev Ecol Evol Syst 39 387-413.

Emlen DJ, Marangelo J, Ball B, Cunningham CW (2005). Diversity in the weapons of sexua selection: horn evolution in the beetle genus Onthophagus (Coleoptera: Scarabaeidae). Evolution 59: 1060-1084.

Fishman L, Kelly AJ, Willis JH (2002). Minor quantitative trait loci underlie floral traits associated with mating system divergence in Mimulus. Evolution 56: 2138-2155.

Gleason JM, Ritchie MG (2004). Do quantitative trait loci (QTL) for a courtship song difference between Drosophila simulans and D. sechellia coincide with candidate genes and intraspecific QTL? Genetics 166: 1303-1311.

Graze RM, Barmina O, Tufts D, Naderi E, Harmon KL, Persianinova M et al. (2007). New candidate genes for sex-comb divergence between Drosophila mauritiana and Drosophila simulans. Genetics 176: 2561-2576.

Groot AT, Staudacher H, Barthel A, Inglis O, Schofl G, Santangelo RG et al. (2013). One quantitative trait locus for intra- and interspecific variation in a sex pheromone. $\mathrm{Mol} E \mathrm{CO}$ 22: $1065-1080$.

Grubbs N, Leach M, Su X, Petrisko T, Rosario JB, Mahaffey JW (2013). New components of Drosophila leg development identified through genome wide association studies. PLoS One 8: e60261.

Guan X, Middlebrooks BW, Alexander S, Wasserman SA (2006). Mutation of TweedleD a member of an unconventional cuticle protein family, alters body shape in Drosophila. Proc Natl Acad Sci USA 103: 16794-16799.

Hall MC, Basten CJ, Willis JH (2006). Pleiotropic quantitative trait loci contribute to population divergence in traits associated with life-history variation in Mimulus guttatus. Genetics 172: 1829-1844.

Hosken DJ, Stockley P (2004). Sexual selection and genital evolution. Trends Ecol Evol 19 87-93.

Huang Y, Erezyilmaz D (2015). The genetics of resistance to Morinda fruit toxin during the postembryonic stages in Drosophila sechellia. G3 (Bethesda) 5: 1973-1981.

Hurtado-Gonzales JL, Gallaher W, Warner A, Polak M (2015). Microscale laser surgery demonstrates the grasping function of the male sex combs in Drosophila melanogaster and Drosophila bipectinata. Ethology 121: 45-56.

Johnson T, Barton N (2005). Theoretical models of selection and mutation on quantitative traits. Philos Trans R Soc Lond B Biol Sci 360: 1411-1425.
King EG, Kislukhin G, Walters KN, Long AD (2014). Using Drosophila melanogaster to identify chemotherapy toxicity genes. Genetics 198: 31-43.

King EG, Macdonald SJ, Long AD (2012a). Properties and power of the Drosophila Synthetic Population Resource for the routine dissection of complex traits. Genetics 191: 935-949.

King EG, Merkes CM, McNeil CL, Hoofer SR, Sen S, Broman KW et al. (2012b), Genetic dissection of a model complex trait using the Drosophila Synthetic Population Resource. Genome Res 22: 1558-1566.

Kislukhin G, King EG, Walters KN, Macdonald SJ, Long AD (2013). The genetic architecture of methotrexate toxicity is similar in Drosophila melanogaster and humans. G3 (Bethesda) 3: 1301-1310.

Kopp A (2011). Drosophila sex combs as a model of evolutionary innovations. Evol Dev 13 : 504-522.

Kopp A, Graze RM, Xu SZ, Carroll SB, Nuzhdin SV (2003). Quantitative trait loci responsible for variation in sexually dimorphic traits in Drosophila melanogaster. Genetics 163: 771-787.

Kopp A, True JR (2002). Evolution of male sexual characters in the oriental Drosophila melanogaster species group. Evol Dev 4: 278-291.

Lewis RA, Kaufman TC, Denell RE, Tallerico P (1980a). Genetic analysis of the Antennapedia gene complex (Ant-C) and adjacent chromosomal regions of Drosophila melanogaster. I. Polytene chromosome segments 84b-D. Genetics 95: 367-381.

Lewis RA, Wakimoto BT, Denell RE, Kaufman TC (1980b). Genetic analysis of the Antennapedia gene complex (Ant-C) and adjacent chromosomal regions of Drosophila melanogaster. II. Polytene chromosome segments 84A-84B1,2. Genetics 95: 383-397.

Lewontin RC (1974). The Genetic Basis of Evolutionary Change. Columbia University Press: New York, NY.

Lexer C, Rosenthal DM, Raymond O, Donovan LA, Rieseberg LH (2005). Genetics of species differences in the wild annual sunflowers, Helianthus annuus and $\mathrm{H}$. petiolaris. Genetics 169: 2225-2239.

Ma Y, Creanga A, Lum L, Beachy PA (2006). Prevalence of off-target effects in Drosophila RNA interference screens. Nature 443: 359-363.

Macdonald SJ, Goldstein DB (1999). A quantitative genetic analysis of male sexual traits distinguishing the sibling species Drosophila simulans and D. sechellia. Genetics 153: 1683-1699.

Mackay TF (2001). Quantitative trait loci in Drosophila. Nat Rev Genet 2: 11-20.

Markow T, Bustoz D, Pitnick S (1996). Sexual selection and a secondary sexual character in two Drosophila species. Animal Behav 52: 759-766.

Marriage TN, King EG, Long AD, Macdonald SJ (2014). Fine-mapping nicotine resistance loci in Drosophila using a multiparent advanced generation inter-cross population. Genetics 198: 45-57.

McIntyre LM, Bono LM, Genissel A, Westerman R, Junk D, Telonis-Scott M et al. (2006). Sex-specific expression of alternative transcripts in Drosophila. Genome Biol 7: R79.

McNeil CL, Bain CL, Macdonald SJ (2011). Multiple quantitative trait loci influence the shape of a male-specific genital structure in Drosophila melanogaster. G3 (Bethesda) 1: 343-351.

Mitchell-Olds T, Willis JH, Goldstein DB (2007). Which evolutionary processes influence natural genetic variation for phenotypic traits? Nat Rev Genet 8: 845-856.

$\mathrm{Ng}$ CS, Kopp A (2008). Sex combs are important for male mating success in Drosophila melanogaster. Behav Genet 38: 195-201.

Norga KK, Gurganus MC, Dilda CL, Yamamoto A, Lyman RF, Patel PH et al. (2003). Quantitative analysis of bristle number in Drosophila mutants identifies genes involved in neural development. Curr Biol 13: 1388-1396.

Nuzhdin SV, Reiwitch SG (2000). Are the same genes responsible for intra- and interspecific variability for sex comb tooth number in Drosophila? Heredity 84 97-102.

Pan Y, Robinett CC, Baker BS (2011). Turning males on: activation of male courtship behavior in Drosophila melanogaster. PLoS One 6: e21144

Parker GA (1979)Sexual selection and sexual conflictln:Blum MS, Blum NA(eds) Sexual Selection and Reproductive Competition in Insects. Academic Press: New York, NY. pp 123-166.

Pinheiro J, Bates D, DebRoy S, Sarkar DTeam RDC (2011). nlme: linear and nonlinear mixed effects models. $R$ package version 31-101.

Polak M, Starmer WT, Wolf LL (2004). Sexual selection for size and symmetry in a diversifying secondary sexual character in Drosophila bipectinata duda (Diptera: Drosophilidae). Evolution 58: 597-607.

Provine WB (1971). The Origins of Theoretical Population Genetics. The University of Chicago Press: Chicago, IL.

Quinn LM, Dickins RA, Coombe M, Hime GR, Bowtell DD, Richardson H (2004). Drosophila Hfp negatively regulates dmyc and stg to inhibit cell proliferation. Development 131: 1411-1423.

Ranz JM, Maurin D, Chan YS, von Grotthuss M, Hillier LW, Roote J et al. (2007). Principles of genome evolution in the Drosophila melanogaster species group. PLoS Biol 5: e152.

Rideout EJ, Dornan AJ, Neville MC, Eadie S, Goodwin SF (2010). Control of sexual differentiation and behavior by the doublesex gene in Drosophila melanogaster. Nat Neurosci 13: 458-466.

Robinett CC, Vaughan AG, Knapp JM, Baker BS (2010). Sex and the single cell. II. There is a time and place for sex. PLOS Biol 8: e1000365.

Sharma MD, Tregenza T, Hosken DJ (2011). Sex combs, allometry, and asymmetry in Drosophila. Biol J Linn Soc 103: 923-934.

Snook RR, Gidaszewski NA, Chapman T, Simmons LW (2013). Sexual selection and the evolution of secondary sexual traits: sex comb evolution in Drosophila. J Evol Biol 26: 912-918. 
Spieth HT (1952). Mating behavior within the genus Drosophila (Diptera). Bull Am Mus Nat Hist 99: 395-474.

St Pierre SE, Galindo MI, Couso JP, Thor S (2002). Control of Drosophila imaginal disc development by rotund and roughened eye: differentially expressed transcripts of the same gene encoding functionally distinct zinc finger proteins. Development 129: 1273-1281.

Tanaka K, Barmina O, Sanders LE, Arbeitman MN, Kopp A (2011). Evolution of sex-specific traits through changes in HOX-dependent doublesex expression. PLoS Biol 9: e1001131.

Tatsuta H, Takano-Shimizu T (2006). Genetic architecture of variation in sex-comb tooth number in Drosophila simulans. Genet Res 87: 93-107.

True JR, Liu JJ, Stam LF, Zeng ZB, Laurie CC (1997). Quantitative genetic analysis of divergence in male secondary sexual traits between Drosophila simulans and Drosophila mauritiana. Evolution 51: 816-832.

Turelli M, Barton NH (2004). Polygenic variation maintained by balancing selection: pleiotropy, sex-dependent allelic effects and G x E interactions. Genetics 166 1053-1079.

Van Buskirk C, Schupbach T (2002). Half pint regulates alternative splice site selection in Drosophila. Dev Cell 2: 343-353.

Wang S, Wagner EJ, Mattox W (2013). Half pint/Puf68 is required for negative regulation of splicing by the SR splicing factor Transformer2. RNA Biol 10: 1396-1406.
Wittkopp PJ, Stewart EE, Arnold LL, Neidert AH, Haerum BK, Thompson EM et al. (2009). Intraspecific polymorphism to interspecific divergence: genetics of pigmentation in Drosophila. Science 326: 540-544.

Xu S (2003). Theoretical basis of the Beavis effect. Genetics 165: 2259-2268.

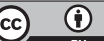

This work is licensed under a Creative Commons Attribution 4.0 International License. The images or other third party material in this article are included in the article's Creative Commons license, unless indicated otherwise in the credit line; if the material is not included under the Creative Commons license, users will need to obtain permission from the license holder to reproduce the material. To view a copy of this license, visit http:// creativecommons.org/licenses/by/4.0/

(C) The Author(s) 2016

Supplementary Information accompanies this paper on Heredity website (http://www.nature.com/hdy) 